\title{
Photic Entrainment of Period Mutant Mice is Predicted from Their Phase Response Curves
}

\author{
Julie S. Pendergast, Rio C. Friday, and Shin Yamazaki \\ Department of Biological Sciences, Vanderbilt University, Nashville, Tennessee, 37235-1634
}

\begin{abstract}
A fundamental property of circadian clocks is that they entrain to environmental cues. The circadian genes, Period1 and Period2, are involved in entrainment of the mammalian circadian system. To investigate the roles of the Period genes in photic entrainment, we constructed phase response curves (PRC) to light pulses for C57BL/6J wild-type, Per1 ${ }^{-I-}$, Per2 ${ }^{-I-}$, and Per $3^{-I-}$ mice and tested whether the PRCs accurately predict entrainment to non-24 light- dark cycles (T-cycles) and constant light (LL). The PRCs of wild-type and $P e r 3^{-1-}$ mice are similar in shape and amplitude and have relatively large delay zones and small advance zones, resulting in successful entrainment to $26 \mathrm{~h} \mathrm{~T}$-cycles (T26), but not T21, with similar phase angles. Per1 ${ }^{-1-}$ mice have a high-amplitude PRC, resulting in entrainment to a broad range of T-cycles. Per2 ${ }^{-l-}$ mice also entrain to a wide range of T-cycles because the advance portion of their PRC is larger than wild types. Period aftereffects following entrainment to T-cycles were similar among all genotypes. We found that the ratio of the advance portion to the delay portion of the PRC accurately predicts the lengthening of the period of the activity rhythm in LL. Wild-type, Per1 ${ }^{-I-}$, and Per $3^{-I-}$ mice had larger delay zones than advance zones and lengthened $(>24 \mathrm{~h})$ periods in LL, whereas Per2 ${ }^{-1-}$ mice had delay and advance zones that were equal in size and no period lengthening in LL. Together, these results demonstrate that PRCs are powerful tools for predicting and understanding photic entrainment of circadian mutant mice.
\end{abstract}

\section{Introduction}

A fundamental property of circadian oscillators is that their rhythms are entrained to environmental cycles so that the internal phase of the clock is a representation of external time (Johnson et al., 2003). Two distinct models have been proposed to describe entrainment of the circadian clock to the light-dark (LD) cycle: the discrete (nonparametric) and the continuous (parametric) models (Aschoff, 1960; Bruce, 1960; Pittendrigh, 1966; Pittendrigh and Daan, 1976). According to the nonparametric model, light at discrete times of day (e.g., dawn and dusk) falls at specific phases of the pacemaker and causes instantaneous phase shifts that are equal to the difference between the period of the rhythm and the period of the LD cycle. Phase response curves (PRC), which are graphic representations of the shifts of a rhythm in response to stimuli given at different phases, have been used to elucidate nonparametric entrainment mechanisms (Daan and Pittendrigh, 1976a; Johnson, 1999). The continuous model posits that changes in light intensity cause phase-specific accelerations or decelerations of the clock period so that it equals that of the environmental LD cycle (Aschoff, 1960; Daan and Pittendrigh, 1976b). Systematic studies investigating the effect of variable light intensities in constant light (LL) on the period of the rhythm support the mechanism of continuous entrainment.

Received May 21, 2010; revised July 1, 2010; accepted July 27, 2010.

This research was supported by National Institutes of Health grants (NS051278 to S.Y. and F32NS061437 to J.S.P.) and by the Vanderbilt University Summer Research Program (to R.C.F.). We thank David Weaver for $m P e r 7^{\text {Idc }-1-}$, $m P e r 2^{I d c-/-}$, and $\mathrm{mPer} 3^{-1-}$ mice. We also thank Karen Gamble for guidance regarding statistical analyses.

Correspondence should be addressed to Shin Yamazaki, Department of Biological Sciences, Vanderbilt University, VU Station B, Box 35-1634, Nashville, TN 37235. E-mail: shin.yamazaki@vanderbilt.edu.

DOI:10.1523/JNEUROSCI.2607-10.2010

Copyright $\odot 2010$ the authors $\quad 0270-6474 / 10 / 3012179-06 \$ 15.00 / 0$
Period (Per)1 and Per2 are the only components of the circadian timekeeping machinery known to acutely respond to environmental signals. Light stimulation induces the expression of Per 1 and Per2, but not Per3, mRNA in the suprachiasmatic nucleus (SCN) (Albrecht et al., 1997; Shearman et al., 1997; Shigeyoshi et al., 1997; Takumi et al., 1998; Zylka et al., 1998). Pretreatment with antisense oligonucleotides to Per1 blocks light-induced phase shifts in activity and glutamateinduced shifts in neuronal activity in SCN explants (Akiyama et al., 1999; Tischkau et al., 2003). In addition, constitutive overexpression of PER1 impairs entrainment of rats to LD cycles (Numano et al., 2006). The phase responsiveness to light is also altered in Per mutant mice (Albrecht et al., 2001; Bae and Weaver, 2003; Spoelstra et al., 2004). Furthermore, novel wheel exposure, which induces nonphotic entrainment, downregulates the expression of Per1 and Per2 mRNAs (Maywood et al., 1999; Yannielli et al., 2002).

To understand the roles of the Per genes in the entrainment mechanism, we obtained complete PRCs to light pulses from C57BL/6J Per mutant mice. These PRCs accurately predict the phase angle of entrainment and responsiveness to LL of each mutant and are powerful tools for understanding the molecular mechanisms of entrainment.

\section{Materials and Methods}

Animals. C57BL/6J mPer1 $1^{l d c-1-}, m P e r 2^{l d c-1-}$, and $m P e r 3^{-1-}$ mice (Shearman et al., 2000; Bae et al., 2001; Pendergast et al., 2009, 2010) were bred and group-housed in a 12-h-light/12-h-dark cycle (12L:12D) and provided food and water ad libitum. Genotype was determined as previously described (Shearman et al., 2000; Bae et al., 2001). Male and female mice were $79.9 \pm 35.4 \mathrm{~d}$ old (mean $\pm \mathrm{SD}$ ) at the beginning of the experiments (the number and sex of mice used for each experiment is provided 

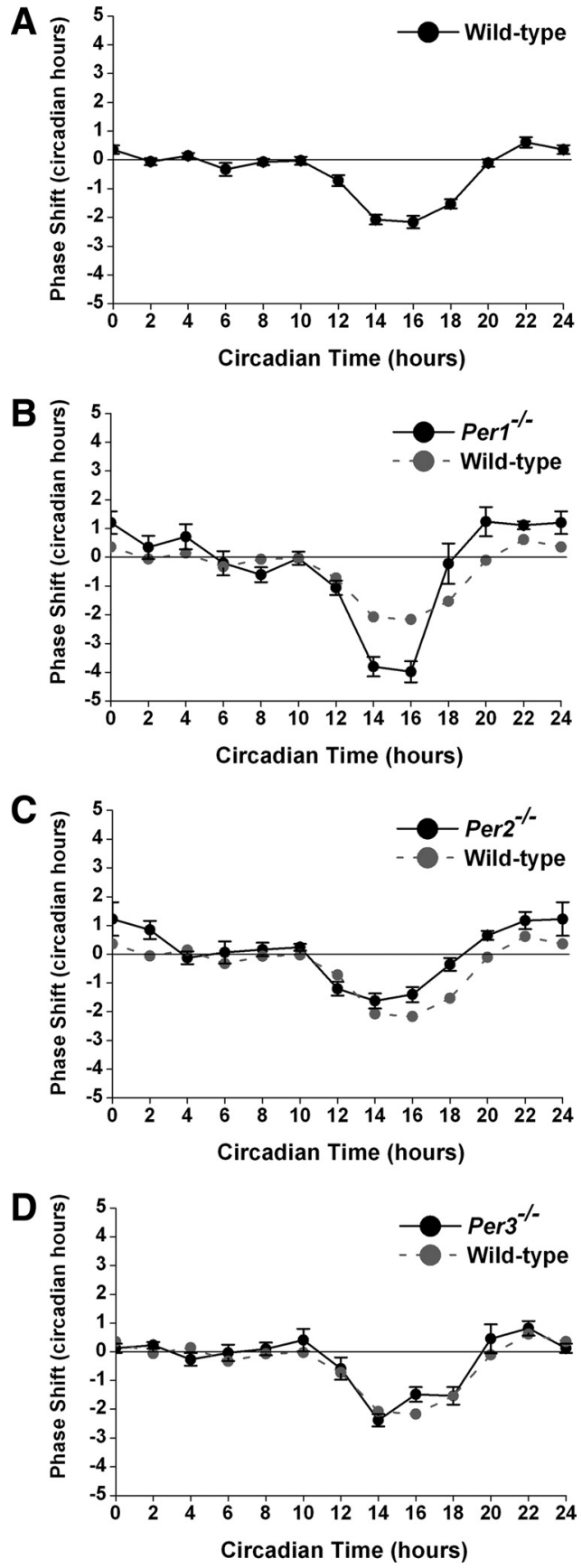

Figure 1. Phase responses to light pulses in wild-type and Period mutant mice. $\boldsymbol{A}-\boldsymbol{D}$, Phase response curves to $15 \mathrm{~min}$ light pulses (150 lux) in wild-type $(\boldsymbol{A}), \operatorname{Per}^{-1-}(\boldsymbol{B}), \operatorname{Per}^{-1-}(\boldsymbol{C})$, and $P$ er3 ${ }^{-1-}(\boldsymbol{D})$ mice. Data are the mean \pm SEM of at least four mice at each time point.

in the supplemental material, available at www.jneurosci.org). Experiments were conducted in accordance with the guidelines of the Vanderbilt University Institutional Animal Care and Use Committee.

Phase response curve experiments. C57BL/6J Period mutant mice are ideal for analyses of light responsiveness because they do not become arrhythmic in constant darkness (DD). Mice were singly housed in a cage $(29.5 \times 11.5 \times 12 \mathrm{~cm})$ with unlimited access to a running wheel (diameter, $11 \mathrm{~cm}$ ) and free-ran for $6 \mathrm{~d}$ in DD. The onset of activity [circadian time (CT) 12] was predicted for each mouse on the following day using linear regression (ClockLab; Actimetrics). At the appropriate CT, the cage was removed from the light-tight box (in the dark using an infrared viewer) and placed in another light-tight box, where the light intensity (white fluorescent bulb, Sylvania Octron 3500K, 32W) was 150 lux at the bottom of the cage. The mouse remained there for $15 \mathrm{~min}$ and then was

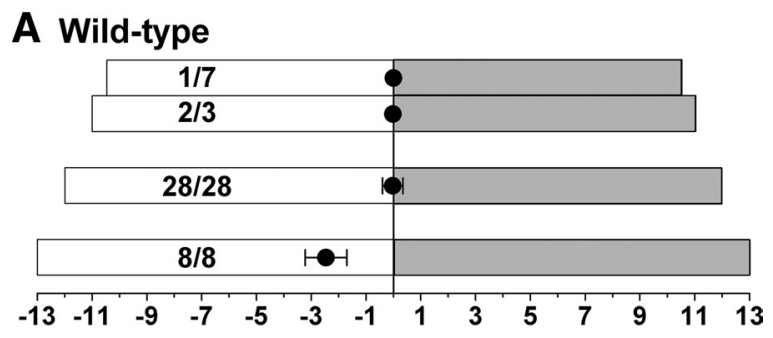

B Per1 ${ }^{-1-}$

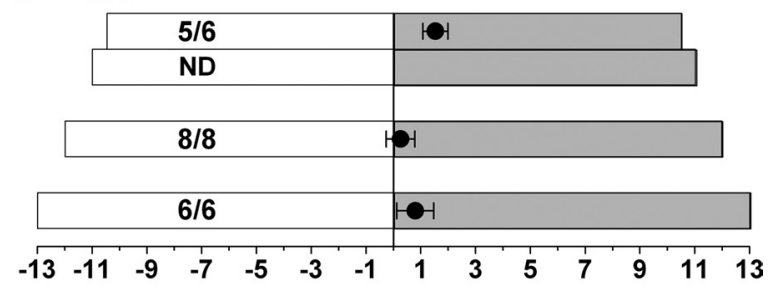

C Per2 ${ }^{-1-}$

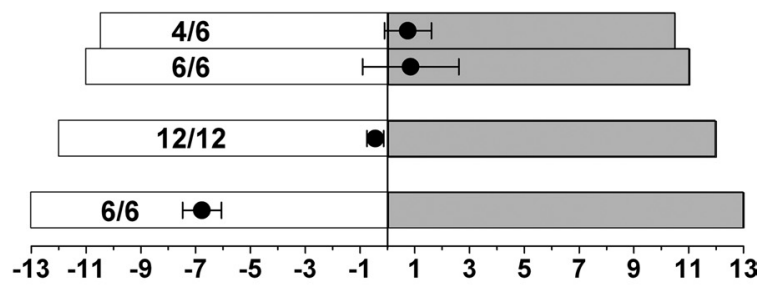

D Per ${ }^{-1-}$

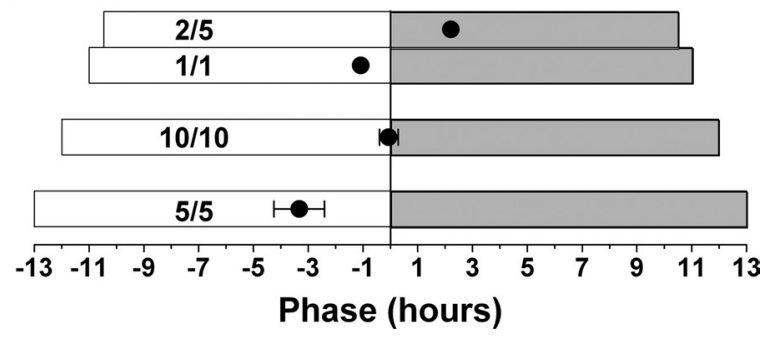

Figure 2. Alterations in phase angles of entrainment to T-cycles in Per1 ${ }^{-1-}$ and Per2 $2^{-1-}$ mice. The length of the LD cycle was gradually reduced (for T21 and T22) or gradually lengthened (for T26) by $1 \mathrm{~h} /$ week. The mice were maintained in the target T-cycle for 2 weeks and then released into $D D$. Naive mice were used for each T-cycle. $\boldsymbol{A}-\boldsymbol{D}$, The phase angles of entrainment (mean \pm SD h) for wild-type $(\boldsymbol{A})$, Per1 $^{-1-}(\boldsymbol{B})$, Per2 ${ }^{-1-}\left(\boldsymbol{C}\right.$, and Per $3^{-1-}(\boldsymbol{D})$ mice were determined by drawing a regression line to activity onset for $1-5$ in $D D$ and then extending the regression line to the last day in LD. Actual time (not circadian time) is plotted. The length of the T-cycle is indicated by the white (lights on) and gray (lights off) boxes. The T24 data were reported previously (Pendergast et al., 2009, 2010). The number of mice that entrained to the T-cycle relative to the total number of mice investigated is reported for each group. Per ${ }^{-1-}$ mice were not assessed in T22 (ND, Not determined).

returned to the box in $\mathrm{DD}$. The mice free-ran for 1 week after the light pulse. One regression line was fit to the onset of activity for $6 \mathrm{~d}$ before the light pulse and the second line was fit to the onset of activity for $6 \mathrm{~d}$ following the light pulse and the phase shift was calculated that accounted for the change in period that occurred with the light pulse. The phase-shift values were combined into $2 \mathrm{~h}$ bins. Mice were given light pulses every 2 weeks. Cages were changed in the intervening week between pulses. Individual mice did not receive multiple light pulses at the same phase. Each mouse received $\sim 6$ light pulses. The PRC bisection test (Kripke et al., 2003) was used to determine the phase of the inflection point and amplitude of the PRCs. KruskalWallis one-way ANOVA on ranks followed by post hoc Dunn's method or the Mann-Whitney rank sum test was used to compare the amplitudes of the PRCs. 
Analysis of wheel-running activity in non-24 light-dark cycles and LL. Mice were singly housed in cages $(33 \times 17 \times 14 \mathrm{~cm})$ with unlimited access to a running wheel (diameter, $11 \mathrm{~cm}$ ), food, and water. The cages were placed in light-tight boxes where the light intensity at the bottom of the cage was 200-300 lux (white fluorescent bulbs, GE F18T8-WW, 18W).

For non-24 light-dark cycles (T-cycle) experiments, the length of the LD cycle was gradually increased from T24 (mice bred and raised in 12L:12D) to T25 (1 week) to T26. Alternatively, the length of the LD cycle was gradually decreased from T24 to T23 (1 week) to T22 (1 week) to T21. Mice were maintained in the target T-cycle for 2 weeks and then released into DD. To determine whether mice entrained to the T-cycle, actograms of wheel-running activity, plotted with a modular tau equal to the T-cycle, were inspected by two independent observers who were blinded to the genotype of the mice. Mice were labeled as entrained if the onset of activity in T21 and T22 or the offset of activity in T26 maintained a stable phase relationship with lights off for the last $7 \mathrm{~d}$ in the T-cycle. The period and phase angle of entrainment were determined only from mice that entrained to the T-cycle. The free-running period was determined by $\chi^{2}$ periodogram for days $1-15$ in DD (ClockLab).

For LL experiments, naive mice were transferred from the breeding room (12L:12D) to the same 12L:12D in the light-tight boxes for at least $4 \mathrm{~d}$, and then released into LL. The free-running period was determined by $\chi^{2}$ periodogram for $10 \mathrm{~d}$ in LL (the rhythm sometimes dissociated into multiple components after $10 \mathrm{~d}$ in LL). Independent $t$ tests (two-tailed) were used to compare the periods in DD with LL except when the data were not normally distributed and then the Mann-Whitney rank sum test was used. The phase angle of entrainment, amplitude $\left(Q_{p}\right)$, and total activity were determined (Pendergast et al., 2009) and the differences between groups were compared by one-way ANOVA.

\section{Results}

\section{Altered phase responses to light pulses in $\mathrm{C} 57 \mathrm{BL} / 6 \mathrm{~J} \mathrm{Per1}{ }^{-1-}$} and Per $2^{-1-}$ mice

We measured the effects of single light pulses on the phase of wheelrunning activity in C57BL/6J wild-type, $\mathrm{Per} 1^{-/-}, \mathrm{Per} 2^{-/-}$, and Per3 ${ }^{-1-}$ mice (Fig. 1; and supplemental Table S1 and Figs. S1, S2, available at www.jneurosci.org as supplemental material). Consistent with previously reported PRCs for C57BL/6J mice (Daan and Pittendrigh, 1976a; Schwartz and Zimmerman, 1990), wildtype mice exhibited a maximum phase delay $(\sim 2 \mathrm{~h})$ at CT16 and a maximum phase advance $(\sim 0.6 \mathrm{~h})$ at CT22 (Fig. $1 A$; supplemental Fig. S1 $A$, available at www.jneurosci.org as supplemental material). The amplitude of the PRC was significantly greater in Per1 ${ }^{-1-}$ mice (44.3) compared with wild-types (19.0; KruskalWallis, $H=18.07, p<0.001$, Dunn's $p<0.05$ ) (Fig. $1 B$; supplemental Fig. S1 $B$, available at www.jneurosci.org as supplemental material). The amplitudes of the Per2 ${ }^{-1-}(22.8)$ and $P e r 3^{-1-}$ (20.2) PRCs did not differ from wild types (Fig. 1C,D; supplemental Fig. S1C,D, available at www.jneurosci.org as supplemental material). The switch from delays to advances was advanced in Per $1^{-1-}$ (CT18.4) and Per2 ${ }^{-1-}$ (CT19.9) mice compared with wild-type (CT21.9) and Per3 ${ }^{-1-}$ mice (CT20.1). In addition, the advance zones of the PRCs of Per1 ${ }^{-/-}$and Per2 ${ }^{-1-}$ mice were elongated compared with wild types.

Wild-type mice had asymmetrical PRCs such that the delay portion was larger than the advance portion (supplemental Fig. S3, available at www.jneurosci.org as supplemental material). The ratio of the advance area to the delay area $(A / D)$ in the PRC for Per $1^{-/-}$mice (0.4) was greater than in wild types (0.1). The areas of the advance and delay portions of the Per $2^{-1-}$ PRC were nearly equal to each other $(\mathrm{A} / \mathrm{D}=0.9) . \operatorname{Per}^{-1-}$ mice had a slightly larger advance zone than wild types $(\mathrm{A} / \mathrm{D}=0.3)$.

\section{Entrainment to T-Cycles is altered in Period mutant mice}

We next examined entrainment of Per mutant mice to LD cycles of variable lengths (T-cycles). Most wild-type mice (6 of 7) did
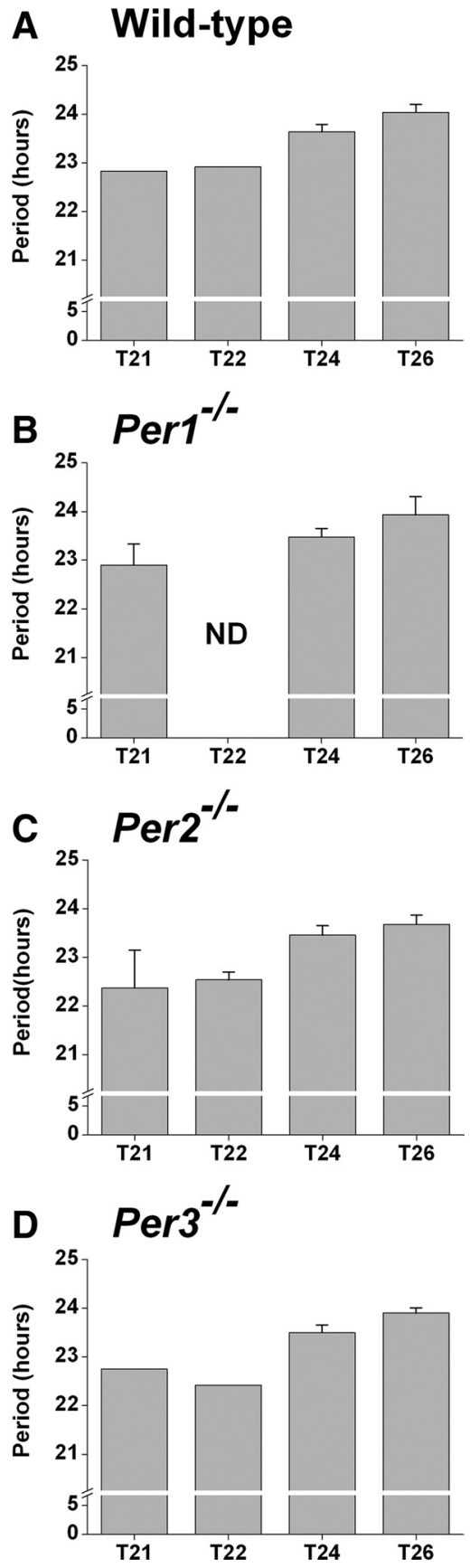

Figure 3. Period aftereffects are not altered in Period mutant mice. After gradually changing the length of the T-cycle, mice were maintained in the target T-cycle for 2 weeks and then released into DD. $\boldsymbol{A}-\boldsymbol{D}$, The free-running periods (mean $\pm \mathrm{SD}$ ) of the wheel-running activity rhythms following entrainment to T21, T22, T24, and T26 in wild-type $(\boldsymbol{A}), \operatorname{Per}^{-1-}(\boldsymbol{B})$, Per2 ${ }^{-1-}(\boldsymbol{C})$, and $\operatorname{Per} 3^{-1-}(\boldsymbol{D})$ mice were determined by $\chi^{2}$ periodogram for $\mathrm{d} 1-15$ in DD. The period was determined only from mice that entrained to the T-cycle (the number of mice that entrained in each group is indicated in Fig. 2). Per $1^{-1-}$ mice were not assessed in T22 (ND, Not determined).

not entrain to T21, but entrained with a phase angle of nearly $0 \mathrm{~h}$ to T22 and T24 (Fig. 2A; supplemental Fig. S4A, available at www.jneurosci.org as supplemental material). In T26, the phase was advanced by $\sim 2.5 \mathrm{~h}$ in wild-type mice (Fig. $2 A$; supplemental Fig. S4D, available at www.jneurosci.org as supplemental material). Most Per ${ }^{-1-}$ mice in T21, T24, and T26 entrained with a phase that was within $\sim 1$ h of lights off (Fig. $2 B$; supplemental Fig. $S 4 B, E$, available at www.jneurosci.org as supplemental material). Although 
the phase of the rhythm was within $\sim 1$ h of lights off in Per2 ${ }^{-1-}$ mice in T21, T22, and $\mathrm{T} 24$, the phase was advanced by $\sim 7 \mathrm{~h}$ in $\mathrm{T} 26$ (Fig. 2C; supplemental Figs. S4C,F, available at www.jneurosci.org as supplemental material). Similar to wild-type mice, the phase of Per3 $3^{-1-}$ mice was within $\sim 1$ h of lights off in T22 and T24, but was advanced by $\sim 3$ $\mathrm{h}$ in T26 (Fig. 2D).

\section{Period aftereffects are not altered in Period mutant mice}

To determine whether period aftereffects or changes in the period as a result of the prior lighting condition were altered in Per mutant mice, we measured the period of the wheel-running activity rhythm in DD following entrainment to T21, T24, or T26. Similar to wild-type mice (Fig. 3A), the period was short in T21 and progressively lengthened in T24 and T26 in Per $1^{-1-}$ (Fig. 3B), Per2 ${ }^{-1-}$ (Fig. 3C), and Per $^{-1-}$ (Fig. 3D) mice. The slopes of linear trend lines fitted to plots of period versus $\mathrm{T}$-cycle were similar between the genotypes (supplemental Fig. S5, available at www.jneurosci.org as supplemental material).

\section{Periods of the activity rhythm are altered in $\mathrm{C} 57 \mathrm{BL} / 6 \mathrm{~J}$}

\section{Per $1^{-/-}$and Per $2^{-/-}$mice in LL}

The free-running periods of the activity rhythms in LL were longer in wild-type $(p<0.001)$ (Fig. $4 A)$, Per $1^{-1-}\left(t_{(16)}=\right.$ $-13.2, p<0.001$ ) (Fig. $4 B$ ), and Per3 ${ }^{-1-}$ $\left(t_{(12)}=-18.0, p<0.001\right)$ (Fig. $\left.4 D\right)$ mice compared with wild-type, Per $1^{-1-}$, and $P e r 3^{-1-}$ mice, respectively, in DD (separate groups of naive mice were assessed in LL or DD) (Fig. $4 E$ ). In contrast, the freerunning periods of Per2 $2^{-l-}$ mice in LL and DD did not differ from each other $\left(t_{(15)}=0.5\right)$ (Fig. $\left.4 C, E\right)$. These data are consistent with previous studies that examined different lines of Per ${ }^{-1-}$ and Per2 ${ }^{-1-}$ mice in LL (Steinlechner et al., 2002; Spoelstra and Daan, 2008). The amplitude $\left(\mathrm{Q}_{\mathrm{p}} ; F_{(3,27)}=0.88, p=0.47\right)$ (supplemental Fig. S6A, available at www.jneurosci.org as supplemental material $)$, total daily activity $\left(F_{(3,31)}=2.19, p=0.11\right)$ (supplemental Fig. S6 B, available at www.jneurosci.org as supplemental material), and phase $\left(F_{(3,31)}=0.49, p=0.69\right)$ (supplemental Fig. S6C, available at www.jneurosci.org as supplemental material) of the activity rhythm in LL did not differ between wild-type, $P e r 1^{-1-}, P e r 2^{-1-}$, and Per $3^{-1-}$ mice.

\section{Discussion}

A notable feature of the PRC of C57BL/6 $\mathrm{Perl}^{-1-}$ mice is its high amplitude, which is reminiscent of the light responsiveness of Clock mutant $(\Delta 19)$ heterozygous mice that have large phase shifts and low-amplitude circadian oscillations in the SCN (Vitaterna et al., 2006). This is consistent with our finding that the $\mathrm{Perl}^{-1-} \mathrm{SCN}$ is arrhythmic or has a low amplitude, irregular rhythm in vitro (Pendergast et al., 2009). The PRC of Per $2^{-1-}$ mice has an elongated advance zone and large phase advances. In addition, $P e r 2^{-I-}$ mice have a phase-advanced PRC, suggesting that the phase relationship between the circadian pacemaker and the onset of the activity rhythm in Per2 $2^{-1-}$ mice is altered. The PRC of Per3 ${ }^{-1-}$ mice is similar to the PRC of wild-type mice. This is not surprising because Per3 expression is not induced in response to light (Takumi et al., 1998; Zylka et al., 1998).

PRCs have been used to make predictions according to the discrete model of entrainment, which posits that each day the clock must be reset by an amount equivalent to the difference between the period of the clock and of the extrinsic cycle. When an animal that has a clock with an endogenous period of $\sim 24 \mathrm{~h}$ is placed in a short 21-h-LD cycle (T21), the clock must advance each day. Therefore, animals with large advance zones in their PRCs are better able to entrain to short T-cycles. The PRCs of wild-type and Per $3^{-1-}$ mice have small advance zones compared with Per $1^{-1-}$ and Per2 ${ }^{-1-}$ mice. As a result, most wild-type mice (6 of 7) did not entrain to T21. Per $3^{-1-}$ mice have a slightly larger advance zone than wild-type mice, which is consistent with our finding that two of five Per $3^{-1-}$ mice were able to entrain to T21. The advance zone is approximately five times larger in $P e r 1^{-1-}$ and Per $2^{-1-}$ mice compared with wild types and the majority of these mice entrained to T21.

Daily delays of the endogenous clock must occur to entrain to a T-cycle longer than the endogenous period. Since the wild-type and Per mutant mice have large delay zones, they all successfully entrained to T26. However, we found that the phase angle of 
entrainment in T26 reflects the relative sizes of the delay regions of the PRCs. The delay zones of wild-type and Per $3^{-1-}$ mice are nearly equivalent and they both entrain with an $\sim 3 \mathrm{~h}$ advanced phase. The delay zone of $\operatorname{Per} 1^{-1-}$ mice is $\sim 1.5$ times larger than wild types, so they are better able to align their endogenous rhythm with the T26 cycle and entrain with a slightly delayed (but close to 0 ) phase angle. Per $2^{-1-}$ mice have a smaller delay region than wild types, making it more difficult for their clock to align with the long T-cycle and they therefore entrain with a $\sim 7 \mathrm{~h}$ advanced phase.

The aforementioned interpretations assume that the periods of the endogenous rhythms of the wild-type and Per mutant SCN are similar to each other. The phase angle of entrainment can be predicted if the endogenous period of the circadian oscillator, the PRC to light pulses, and the exogenous T-cycle are known. In general, the shorter the endogenous period of the circadian oscillator (typically measured by releasing the animal into DD) relative to the length of the T-cycle, the more advanced the phase of entrainment will be. However, determining the in vivo period of the circadian oscillators of C57BL/6 $\mathrm{Per}^{-1-}$ and Per2 ${ }^{-1-}$ mice is not clear cut. Although the periods of the activity rhythms in DD are nearly identical between wild-type and Per mutant mice, the endogenous periods of $\mathrm{Per} 1^{-1-}$ and $\mathrm{Per} 2^{-1-} \mathrm{SCN}$ are different from wild types (i.e., $P e r 1^{-1-}$ SCN are arrhythmic and Per $2^{-1-}$ SCN have a 1.5 h shorter period than wild types) (Pendergast et al., 2009, 2010). Therefore, we cannot definitively report the period of the circadian oscillator in these mice, nor do we understand how this issue may affect the interpretation of our data. However, it is possible that the advanced $(\sim 7 \mathrm{~h})$ phase angle of entrainment of Per $2^{-1-}$ mice in T26 could reflect the short endogenous period of their SCN in vitro.

Although the discrete model of entrainment and the PRC is successful in predicting responses to short light pulses, the effects of continuous exposure to light must take into account the model of continuous entrainment. According to the continuous model, the period of the circadian clock slows down or speeds up depending on the light intensity at specific phases of the oscillation (Aschoff, 1960). The angular velocity of the oscillator, represented as a velocity response curve (VRC), is estimated from the PRC, resulting in two curves that are similar to each other in shape and amplitude (Daan and Pittendrigh, 1976b; Taylor et al., 2010). Furthermore, the shapes of both the VRC and PRC correlate with species' differences in the length of the period of the activity rhythm in LL such that an animal with a large delay region and relatively smaller advance portion (small A/D ratio) will have a greater increase in period in LL than an animal with a smaller delay region relative to the advance region (large A/D ratio) (Daan and Pittendrigh, 1976b). For example, since the period accelerates during the advance portions of the PRC and decelerates during the delay portions, an animal with $\mathrm{A}>\mathrm{D}$ will have more acceleration than deceleration and therefore a shorter period in LL than an animal with $\mathrm{D}>\mathrm{A}$. We find that the shapes of the Per mutant PRCs correlate with the lengthening of their periods in LL compared with DD. The A/D ratio is small in wildtype mice, resulting in a $\sim 1.5 \mathrm{~h}$ lengthening of their period in LL; $\mathrm{Per}^{-1-}$ mice have an intermediate A/D ratio and an intermediate lengthening of their period in LL; and the A/D ratio is $\sim 1$ in Per $2^{-1-}$ mice (i.e., the advance and delay portions are equal to each other) and they have no period lengthening in LL compared with DD. Per1 $1^{-1-}$ mice have nearly twice as much delay as advance portions in their PRC, consistent with the lengthening of their period in LL compared with DD. However, based only on their A/D ratio, we predicted their period in LL would be similar to wild types. Instead, the period of the activity rhythm in Per $1^{-1-}$ mice in LL is $\sim 2 \mathrm{~h}$ longer than in wild types. Factors such as altered sensitivity to light may contribute to the phenotype of Per $1^{-1-}$ mice in LL.

This study demonstrates that the range of entrainment, the relative phase angles of entrainment to T-cycles, and circadian behavior in LL can be predicted from complete PRCs to light pulses from circadian mutant mice. It is important to note that features of mutant PRCs, such as phase-shifts of the PRC, changes in amplitude, and elongation of advance or delay zones, can only be detected from full PRCs. Therefore, complete PRCs are powerful tools for detecting alterations in photic entrainment in circadian mutant mice.

\section{References}

Akiyama M, Kouzu Y, Takahashi S, Wakamatsu H, Moriya T, Maetani M, Watanabe S, Tei H, Sakaki Y, Shibata S (1999) Inhibition of light- or glutamate-induced mPer1 expression represses the phase shifts into the mouse circadian locomotor and suprachiasmatic firing rhythms. J Neurosci 19:1115-1121.

Albrecht U, Sun ZS, Eichele G, Lee CC (1997) A differential response of two putative mammalian circadian regulators, mper 1 and mper2, to light. Cell 91:1055-1064.

Albrecht U, Zheng B, Larkin D, Sun ZS, Lee CC (2001) MPer1 and mper2 are essential for normal resetting of the circadian clock. J Biol Rhythms 16:100-104.

Aschoff J (1960) Exogenous and endogenous components in circadian rhythms. Cold Spring Harb Symp Quant Biol 25:11-28.

Bae K, Weaver DR (2003) Light-induced phase shifts in mice lacking mPER1 or mPER2. J Biol Rhythms 18:123-133.

Bae K, Jin X, Maywood ES, Hastings MH, Reppert SM, Weaver DR (2001) Differential functions of mPer1, mPer2, and mPer3 in the SCN circadian clock. Neuron 30:525-536.

Bruce VG (1960) Environmental entrainment of circadian rhythms. In Biological clocks, pp 29-48. Cold Spring Harbor, NY: Cold Spring Harbor Laboratory.

Daan S, Pittendrigh CS (1976a) A functional analysis of circadian pacemakers in nocturnal rodents. II. The variability of phase response curves. J Comp Physiol 106:253-266.

Daan S, Pittendrigh CS (1976b) A functional analysis of circadian oscillators in nocturnal rodents. III. Heavy water and constant light: homeostasis of frequency? J Comp Physiol 106:267-290.

Johnson CH (1999) Forty years of PRCs: what have we learned? Chronobiol Int 16:711-743.

Johnson CH, Elliott JA, Foster R (2003) Entrainment of circadian programs. Chronobiol Int 20:741-774.

Kripke DF, Clopton P, Marler MR, Youngstedt SD, Elliott JA (2003) PRC bisection tests. Chronobiol Int 20:1117-1123.

Maywood ES, Mrosovsky N, Field MD, Hastings MH (1999) Rapid downregulation of mammalian period genes during behavioral resetting of the circadian clock. Proc Natl Acad Sci U S A 96:15211-15216.

Numano R, Yamazaki S, Umeda N, Samura T, Sujino M, Takahashi R, Ueda M, Mori A, Yamada K, Sakaki Y, Inouye ST, Menaker M, Tei H (2006) Constitutive expression of the Period1 gene impairs behavioral and molecular circadian rhythms. Proc Natl Acad Sci U S A 103:3716-3721.

Pendergast JS, Friday RC, Yamazaki S (2009) Endogenous rhythms in period1 mutant suprachiasmatic nuclei in vitro do not represent circadian behavior. J Neurosci 29:14681-14686.

Pendergast JS, Friday RC, Yamazaki S (2010) Distinct functions of Period2 and Period 3 in the mouse circadian system revealed by in vitro analysis. PLoS One 5:e8552.

Pittendrigh CS (1966) The circadian oscillation in Drosophila pseudoobscura pupae: a model for the photoperiodic clock. Z Pflanzenphysiol 54:275-307.

Pittendrigh CS, Daan S (1976) A functional analysis of circadian pacemakers in nocturnal rodents. IV. Entrainment: pacemaker as clock. J Comp Physiol 106:291-331.

Schwartz WJ, Zimmerman P (1990) Circadian timekeeping in BALB/c and C57BL/6 inbred mouse strains. J Neurosci 10:3685-3694.

Shearman LP, Zylka MJ, Weaver DR, Kolakowski LF Jr, Reppert SM (1997) 
Two period homologs: circadian expression and photic regulation in the suprachiasmatic nuclei. Neuron 19:1261-1269.

Shearman LP, Jin X, Lee C, Reppert SM, Weaver DR (2000) Targeted disruption of the mPer3 gene: subtle effects on circadian clock function. Mol Cell Biol 20:6269-6275.

Shigeyoshi Y, Taguchi K, Yamamoto S, Takekida S, Yan L, Tei H, Moriya T, Shibata S, Loros JJ, Dunlap JC, Okamura H (1997) Light-induced resetting of a mammalian circadian clock is associated with rapid induction of the mPer1 transcript. Cell 91:1043-1053.

Spoelstra K, Daan S (2008) Effects of constant light on circadian rhythmicity in mice lacking functional cry genes: dissimilar from per mutants. J Comp Physiol A Neuroethol Sens Neural Behav Physiol 194:235-242.

Spoelstra K, Albrecht U, van der Horst GT, Brauer V, Daan S (2004) Phase responses to light pulses in mice lacking functional per or cry genes. J Biol Rhythms 19:518-529.

Steinlechner S, Jacobmeier B, Scherbarth F, Dernbach H, Kruse F, Albrecht U (2002) Robust circadian rhythmicity of Per1 and Per2 mutant mice in constant light, and dynamics of Per1 and Per2 gene expression under long and short photoperiods. J Biol Rhythms 17:202-209.

Takumi T, Taguchi K, Miyake S, Sakakida Y, Takashima N, Matsubara C, Maebayashi Y, Okumura K, Takekida S, Yamamoto S, Yagita K, Yan L,
Young MW, Okamura H (1998) A light-independent oscillatory gene $m P e r 3$ in mouse SCN and OVLT. EMBO J 17:4753-4759.

Taylor SR, Webb AB, Smith KS, Petzold LR, Doyle FJ 3rd (2010) Velocity response curves support the role of continuous entrainment in circadian clocks. J Biol Rhythms 25:138-149.

Tischkau SA, Mitchell JW, Tyan SH, Buchanan GF, Gillette MU (2003) $\mathrm{Ca} 2+/ \mathrm{cAMP}$ response element-binding protein (CREB)-dependent activation of Per1 is required for light-induced signaling in the suprachiasmatic nucleus circadian clock. J Biol Chem 278:718-723.

Vitaterna MH, Ko CH, Chang AM, Buhr ED, Fruechte EM, Schook A, Antoch MP, Turek FW, Takahashi JS (2006) The mouse Clock mutation reduces circadian pacemaker amplitude and enhances efficacy of resetting stimuli and phase-response curve amplitude. Proc Natl Acad Sci U S A 103:9327-9332.

Yannielli PC, McKinley Brewer J, Harrington ME (2002) Is novel wheel inhibition of per 1 and per 2 expression linked to phase shift occurrence? Neuroscience 112:677-685.

Zylka MJ, Shearman LP, Weaver DR, Reppert SM (1998) Three period homologs in mammals: differential light responses in the suprachiasmatic circadian clock and oscillating transcripts outside of brain. Neuron 20: 1103-1110. 